\title{
Use of Ultrasound Investigation in the Treatment Plan of Apical Surgery Relapse
}

\author{
Anida Maria Băbțan ${ }^{1}$, Bianca Adina Boșca ${ }^{2 *}$, Nausica Bianca Petrescu ${ }^{1}$, Codruța Ioana Mirică ${ }^{1}$, \\ Maria Crișan $^{2}$, Radu Septimiu Câmpian ${ }^{1}$ and Aranka Ilea ${ }^{1}$ \\ ${ }^{1}$ Department of Oral Rehabilitation, Oral Health and Dental Office Management, Faculty of Dentistry, "Iuliu Haţieganu" \\ University of Medicine and Pharmacy Cluj-Napoca, Romania
}

${ }^{2}$ Department of Histology, Faculty of Medicine, "Iuliu Haţieganu" University of Medicine and Pharmacy Cluj-Napoca, Romania

*Corresponding author: Bianca Adina Boșca, Department of Histology, Faculty of Medicine, "Iuliu Haţieganu” University of

Medicine and Pharmacy Cluj-Napoca, Romania

\section{ARTICLE INFO}

Received: 㓞 June 06, 2019

Published: 蔧 June 19, 2019

Citation: Băbțan AM, Boșca AB, Petrescu BN, Mirică CI, Crișan M, Câmpian RS, Ilea A. Use of Ultrasound Investigation in the Treatment Plan of Apical Surgery Relapse. Biomed J Sci \& Tech Res 19(1)2019. BJSTR. MS.ID.003230.

\begin{abstract}
Consecutive to endodontic treatment, the periapical infection, either acute or chronic, is a frequent disease in oral pathology. The two clinical features of periapical infection are granulomatous and cystic, characterized by bone loss, root resorption and impairment of tooth implantation. In current practice, the diagnosis of bone resorption is established using bidimensional and tridimensional radiological examinations. The present case report presents the fibrous exophytic wound healing as a rare complication of apicoectomy and shows the advantages of using ultrasonography in determining the size and nature of maxillary mucosa exophytic structures.
\end{abstract}

Keywords: Endodontic Treatment; Periapical Periodontitis; Ultrasonography; Fibrous Healing; Histopathology

Abbreviations: Lipopolysaccharides (LPS); Lipoteichoic Acid (LTA); Magnetic Resonance Imaging (MRI); Computer Tomography (CT); Cone-Beam Computer Tomography (CBCT)

\section{Introduction}

Among the determining factors of chronical periodontitis is listed bacterial microbiota, especially Gram-negative bacteria that induce bone resorption due to lipopolysaccharides (LPS) and Gram-positive bacteria that damage the dental pulp and the periapical tissues through the lipoteichoic acid (LTA) [1]. The treatment of apical periodontitis is performed by mechanical instrumentation and thorough irrigation with antimicrobial substances, followed by the sealing of the root canals. Although the endodontic field seems to emerge with more effective substances used for disinfection and mechanical instrumentation to improve the clinical outcome, the residual endotoxins in the root canals lead to relapse or to complications [2]. The complications such as periapical periodontitis is treated by root-end surgery consisting in the removal of the tooth's apex, followed by retrograde obturation. The association of an oral antibiotic such as ciprofloxacin, ofloxacin or azithromycin is more efficient in the eradication of the microbial infection compared to large-spectrum antibiotics such as penicillin [3]. Cystic periapical infections are less likely to heal after endodontic treatment due to the fact they are self-sustaining even in the absence of the microbial infection [4].

Complications after endodontic treatment mainly appear as a consequence of forwarding beyond the apex the gutta-percha, root-filling material, or other foreign bodies, such as paper points, amalgam, endomethasone, calcium salts [4]. After establishing the diagnosis of the periapical pathology based on clinical and radiological examination (bidimensional radiographies, tridimensional Computer Tomography- CT, Magnetic Resonance Imaging-MRI or Ultrasonography), the histological examination of the biopsy specimens is useful to exclude a malignancy. Rud \& Moller presented a radiographical classification of the periapical tissue after surgery, recommending a minimum of one-year follow-up, ranging from Group 1- complete healing, to Group 4unsatisfactory healing- failed intervention [5].

\section{Case Report}

A 42-year-old male referred two years ago to Oral Rehabilitation, Oral Health and Dental Office Management Department, accusing 
pain at the upper left canine tooth (2.3). Radiographical examination showed an inappropriate endodontic treatment, enlargement of the periapical periodontal space, resorption of periapical cortical bone corresponding to the tooth 2.3. The diagnosis of chronic periodontitis was set and apicoectomy followed by retrograde filling was performed. At one-year follow-up, the patient noticed an excrescence in the maxillary vestibule at the periapical level of tooth 2.3. Clinical examination revealed a $3 / 4 \mathrm{~mm}$ nodular-shaped fibrous structure in the periapical bone cavity post-apicoectomy, extended submucosal in vestibule, $3-5 \mathrm{~mm}$ mesial and distal to tooth 2.3. Retroalveolar radiography at 1-year follow-up showed osteoid healing and no evidence of periapical recidiva (Figure1). After clinical and paraclinical investigation, the diagnosis of vestibular fibroma was set. In locoregional anesthesia (Articain $1 / 100000$ ) the excision of the specimen was performed followed by apicoectomy and apical curettage.

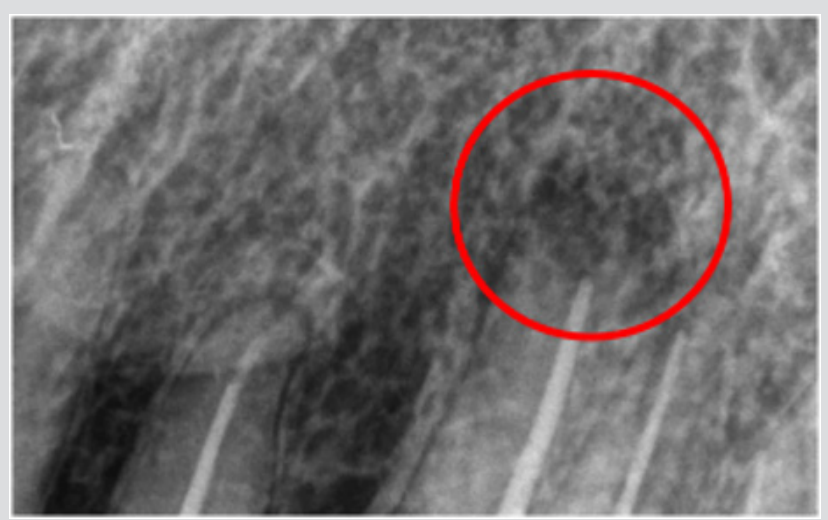

Figure 1: The periapical radiography of upper left canine; 1 year follow-up reveals the osteoid healing (red circle) of the post apicoectomy situs.

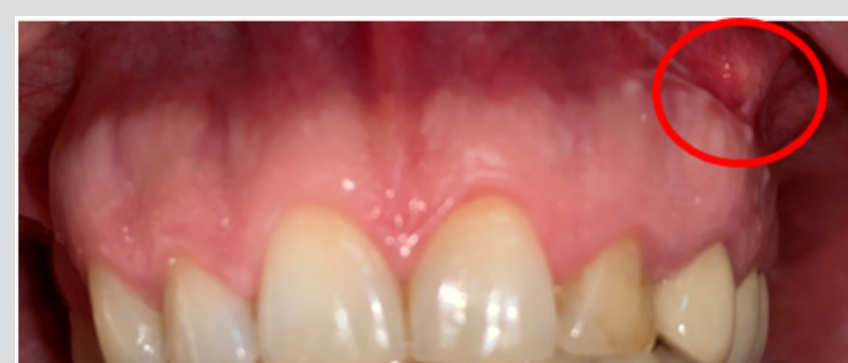

Figure 2: Clinical examination shows a round-shaped fibrous pseudotumoral mass, with slightly peripheral swelling at the upper left canine.

The tissue specimen was sent for histopathological examination and the result showed hyalinized connective tissue, lymphoplasmocitary and macrophagic inflammatory permeation, and microhemorrhage. One year after the second intervention, the patient reported swelling and tumescence at the postsurgical situs, accompanied by inflammation of the surrounding tissues. Clinical examination (Figure 2) showed, on the vestibular fixed-mobile mucosa corresponding to tooth 2.3 , a reddish pseudotumor mass adherent to the surrounding tissues, extended anteriorly, superiorly and mesially, with no inflammatory associated symptoms.

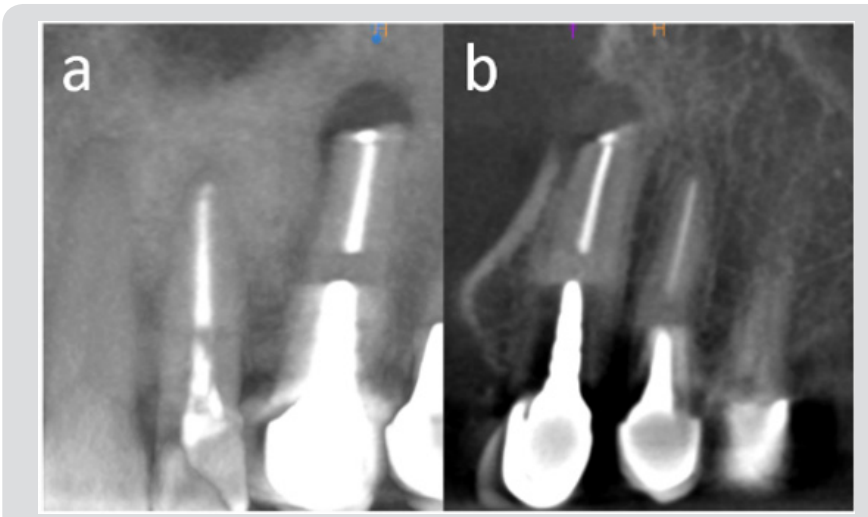

Figure 3: CBCT examination. Coronal (a) and sagittal (b) sections evidence buccal cortical resorption at periapical level of upper left canine and the extension of the resorbtive process along the mesial side of the tooth.

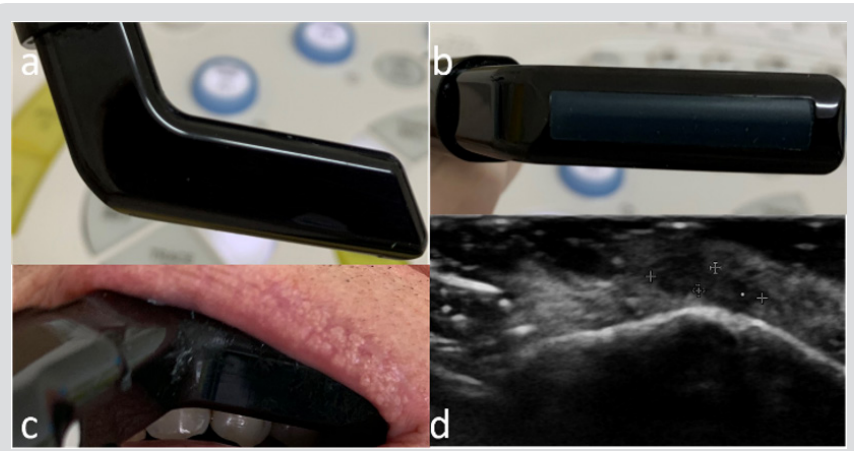

Figure 4: Intraorally ultrasonographic image recorded with a EUP-O54J linear hockey stick transducer (Hitachi) (a - lateral view and b - inferior view, c - intraorally recording approach) showed a $6 / 1.5 \mathrm{~mm}$ diameter hypoechogenic structure (d) at the 2.3 apex level, with extension of hypoechogenicity to the medial and superior maxillary vestibule.

Cone-Beam Computer Tomography (CBCT) showed a round periapical radiolucency at the apex of tooth 2.3 and the resorption of the afferent buccal bone (Figure 3). Ultrasound investigation was performed using a EUP-054J hockey stick linear transducer with a $25 \mathrm{~mm}$ width scan (frequency 7-13 MHz, Hitachi Noblus, Arrieta). Ultrasound imaging (Figure 4) showed a $6 / 1.5 \mathrm{~mm}$ diameter hypoechogenic structure at the apex of tooth 2.3 , with a more intense hypoechogenic extension to the medial and superior maxillary vestibule. Intraoral EcoDoppler ultrasonography (Figure 5) revealed the avascular characteristics of the apical fibrous mass and the blood flow present only on the peripheral area of the fibromatous appearance. Giving the clinical features and ultrasonography investigation, the diagnosis of vestibular fibroma was set. In local anesthesia (Articain 1/100000), the detachment and excision of the fibrous structure from the vestibular mucosa were performed (Figure 6). Due to the structure's adherences, the periosteum was also removed (Figure7). Curettage of the previous 
surgery situs and flap suture were carried out (Figure 8). The harvested specimen was sent for histopathological examination and revealed the presence of connective tissue with extensive areas of hyalinization and minimal lymphocytic inflammatory infiltrate (scarring changes).

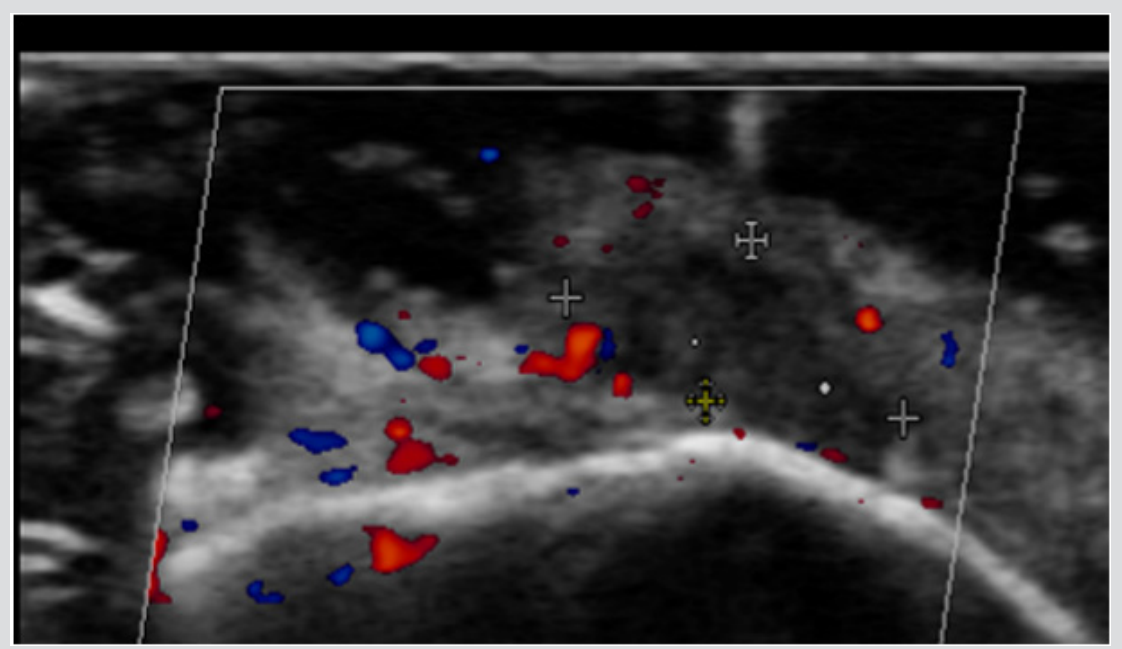

Figure 5: Intraoral EcoDoppler ultrasonography recorded with a EUP-O54J hockey stick linear transducer (Hitachi) demonstrated the avascular characteristic of the apical fibrous structure and its mesial extension.

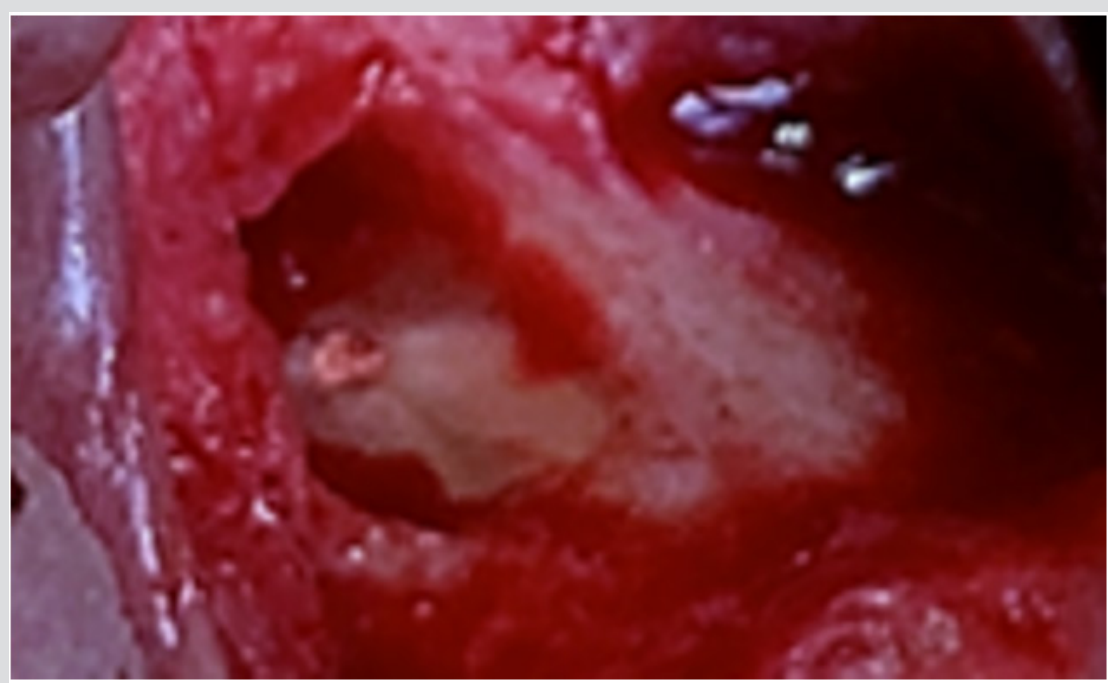

Figure 6: Intraoperatory image showing apicoectomy situs after the excision of the fibrous structure and curettage.

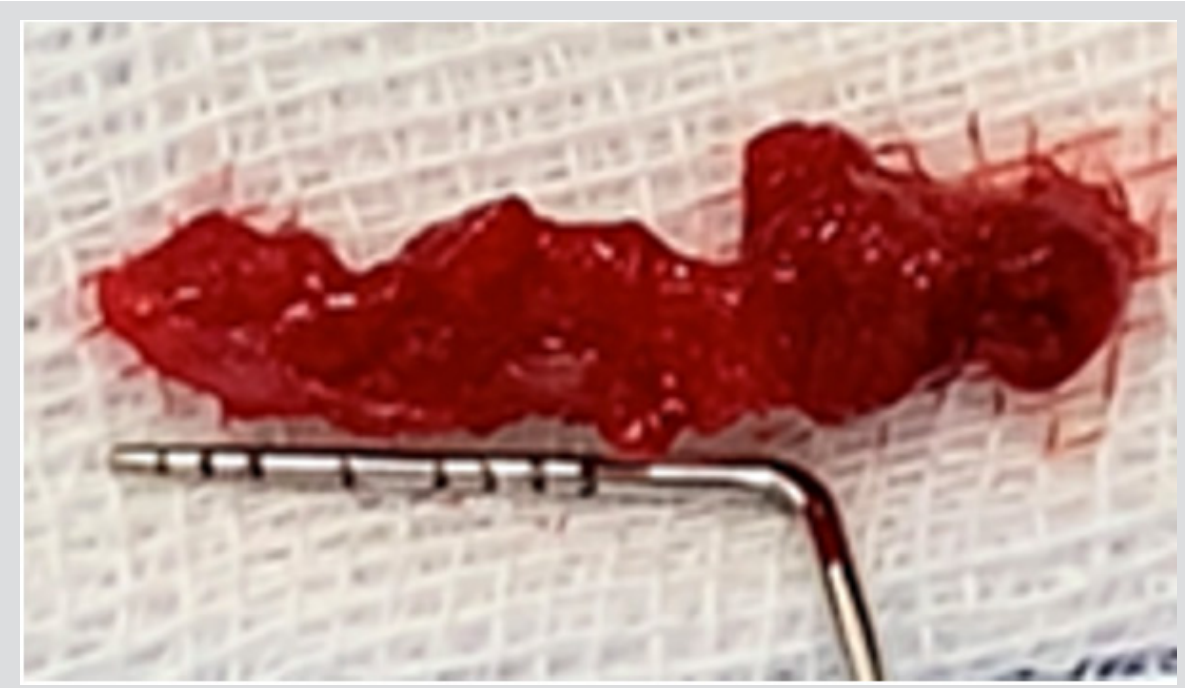

Figure 7: The excised specimen prepared for histopathological investigation. 


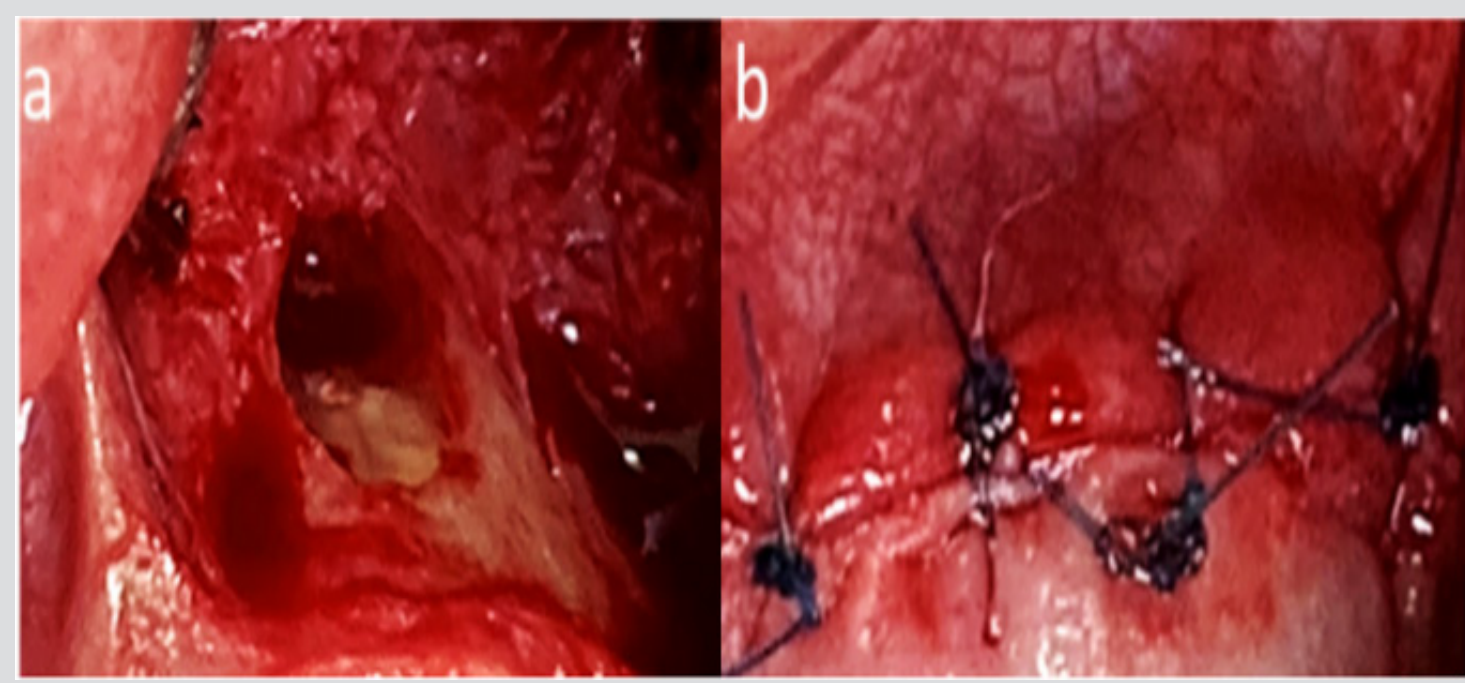

Figure 8: The radicular surface of tooth 2.3 showing apex sealing and removal of all pathological tissues (a) and flap suture (b).

\section{Discussion}

Literature states that regarding apical periodontitis, the specimens sent to histopathogical examination are mostly granulomatous inflammation (due to foreign bodies), benign central giant-cell granulomas, fibro-osseous dysplasia, odontogenic keratocyst and periapical pathologies caused by infections (actinomycosis, histoplasmosis) [6]. Over the time, more accurate radiological methods such as MRI, CT and CBCT were used to investigate the periapical pathologies, especially cysts and granulomas, [7-9]. These methods are very useful in revealing bone resorption and soft tissue changes. In our case, after the first and second surgical interventions, a fibrous healing of both of the periapical bone and vestibular mucosa of the surgical situs were observed. The ultrasound color Doppler investigation showed no vascularization of the exophytic structure. This might be due to a fibroblastic hyperplasia induced by the healing process itself and the proinflammatory mechanisms involved. In dentistry, ultrasonography has been used to diagnose periapical cysts and granulomas, either as primary pathology or consecutive to endodontic treatment, and the postoperative healing. Ultrasonography has the advantage of providing in a nonionizing manner, the size and nature of the analyzed structures [10-12]. In the presented case, the excised specimen had a larger size in mesiodistal direction compared with the ultrasound measurements, but with a similar shape in frontal section. The mesial extension showed a higher hypoechogenicity compared with the exophytic fibrous structure adjacent to tooth 2.3 , which might be due to the dense fibrous hyperplasic and hyalinized structure of the tissue.

\section{Conclusion}

Ultrasonography is a non-invasive, nonionizing, painless, repetitive and real-time imaging method that can be used for examinations both the soft and the hard tissues. It could be taken into consideration as a more suitable investigation compared with the current radiological assays, due to the measurement and Doppler functions, which allows the assessment of the vascularization pattern of oral cavity lesions and periapical pathological tissues. This represents an advantage in setting a treatment protocol, foreseeing possible complications and their management. The assessment of ultrasonography is an attractive and useful approach and should be considered to be implemented as an area of expertise in dentistry and oral surgery practice.

\section{Acknowledgement}

This study was supported by "Iuliu Haţieganu" University of Medicine and Pharmacy Cluj-Napoca, PhD Grant no 3999/01.10.2016, and partially by the COFUND-ERA-HDHL ERANET Project, European and International Cooperation Subprogram 3.2 - Horizon 2020, PNCDI III Program - Biomarkers for Nutrition and Health - "Innovative technological approaches for validation of salivary AGEs as novel biomarkers in evaluation of risk factors in diet-related diseases", no 25/1.09.2017.

\section{References}

1. Barbosa Ribeiro M, D Jesus Soares A, Zaia A, Ferraz C, Almeida J, et al. (2016) Quantification of lipoteichoic acid contents and cultivable bacteria at the different phases of the endodontic retreatment J Endod 42(4): 552-556.

2. Gomes BPF de A, Herrera DR (2018) Etiologic role of root canal infection in apical periodontitis and its relationship with clinical symptomatology Braz. Oral Res 32(1): 82-110.

3. Narita M, Shibahara T, Takano N, Fujii R, Okuda K, et al. (2016) Antimicrobial Susceptibility of Microorganisms Isolated from Periapical Periodontitis Lesions Bull Tokyo Dent Coll 57(3): 133-142.

4. Nair P (2006) On the Causes of Persistent Apical Periodontitis-a Review. Int Endod J 39(4): 249-81.

5. Rud J, Andreasen J, Jensen J (1972) Radiographic criteria for the assessment of healing after endodontic surgery. Int J Oral Surg 4(1): 195-214.

6. Peters E, Lau M (2003) Histopathologic examination to confirm diagnosis of periapical lesions: a review. J Can Dent Assoc 69(9): 598-600.

7. Juerchott A, Pfefferle T, Flechtenmacher C, Mente J, Bendszus M, et al. (2018) Differentiation of periapical granulomas and cysts by using dental MRI: a pilot study Int J Oral Sci 10(2): 17. 
8. Kocak N, Alpoz E, Boyacioglu H (2018) Evaluation of the effect of apical lesion on mucosal thickening and thickness of apical bone using limited cone-beam computed tomography Niger. J Clin Pract 21(8): 954-959.

9. Daviet Noual V, Ejeil AL, Gossiome C, Moreau N, Salmon B, et al. (2017) Differentiating early stage florid osseous dysplasia from periapical endodontic lesions: A radiological-based diagnostic algorithm. BMC Oral Health 17(1): 1-8.

10. Goel S, Raju MS, Rastogi R, Dayashankara Rao Jingade Krishnojira, Swati Gupta, et al. (2011) Ultrasonography with color Doppler and power

\section{ISSN: 2574-1241}

DOI: 10.26717/BJSTR.2019.19.003230

Carmelo Buttà. Biomed J Sci \& Tech Res

(C) This work is licensed under Creative

Submission Link: https://biomedres.us/submit-manuscript.php
Doppler in the diagnosis of periapical lesions Indian. J Radiol Imaging 21(4): 279.

11. Marotti J, Heger S, Tinschert J, Tortamano P, Chuembou F, et al. (2013) Recent advances of ultrasound imaging in dentistry-a review of the literature Oral Surg. Oral Med Oral Pathol Oral Radiol 115(6): 819-832.

12. Prince CN, Annapurna CS, Sivaraj S, Ali I (2012) Ultrasound imaging in the diagnosis of periapical lesions. J Pharm Bioallied Sci 4(6): 369.

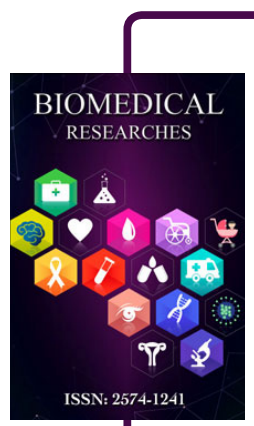

Assets of Publishing with us

- Global archiving of articles

- Immediate, unrestricted online access

- Rigorous Peer Review Process

- Authors Retain Copyrights

- Unique DOI for all articles

https://biomedres.us/ 